\title{
Nonclassical Regimes of Wave Diffraction in Combustible Mixtures
}

\author{
A. A. Vasil'ev, ${ }^{1}$ M. S. Drozdov, ${ }^{2}$ \\ and S. G. Khidirov ${ }^{2}$
}

UDC 534.222.2+536.46+661.215.1

\begin{abstract}
Translated from Fizika Goreniya i Vzryva, Vol. 42, No. 6, pp. 137-143, November-December, 2006. Original article submitted January 1, 2006.

Experimental and numerical results of investigating the diffraction of combustion and detonation waves, including the diffraction in unsteady deflagration-to-detonation transition regimes, are presented.

Key words: combustion, detonation, deflagration-to-detonation transition, wave diffraction, critical diffraction diameter, criterion of diffraction re-initiation.
\end{abstract}

\section{INTRODUCTION}

Propagation of a detonation wave (DW) in the region with a sudden change in the charge size [e.g., if the DW passes from a tube into a half-space $(\nu=3)$ or from a narrow to a wider channel $(\nu=2)]$ is accompanied by a complicated unsteady flow. The generic term for this phenomenon is diffraction. If the detonation wave propagates in a combustible gas mixture, diffraction is accompanied by disintegration and restoration of the ordered cellular structure of the multifront DW.

Previous investigations, beginning from the pioneering works [1-5] performed in the classical formulation (suddenly expanding gas charge, diffraction angle $\alpha=90^{\circ}$ ), revealed the existence of two qualitatively different types of wave propagation after DW diffraction, depending on the relation between the characteristic size of the initial charge and the physical and chemical scales of the explosive mixture: 1) initially selfsustaining DW decays after the beginning of interaction with the expanding corner and degenerates into an unsteady system including a decaying shock wave (SW) and an accompanying turbulent flame; 2) re-initiation of the expanding DW is observed after the beginning of interaction (Fig. 1). The characteristic scale of the gas charge can be, e.g., the tube diameter $d$ in the case of

\footnotetext{
${ }^{1}$ Lavrent'ev Institute of Hydrodynamics, Siberian Division, Russian Academy of Sciences, Novosibirsk 630090; gasdet@hydro.nsc.ru.

${ }^{2}$ Department of the Institute of Power-Engineering Problems of Chemical Physics, Russian Academy of Sciences, Chernogolovka 142432; drozdov@binep.ac.ru.
}

DW transformation into a quasi-spherical wave or the channel width $l$ in the case of DW transformation into a quasi-cylindrical wave; the characteristic size of the mixture is the cell size $a$ of the multifront DW.

Based on the first results of studying DW diffraction, the following relation was proposed as a criterion for estimating the critical diameter $d_{* *}[3-5]$ :

$$
d_{* *}=13 a=\text { const. }
$$

The diameter being equal to the critical value $d_{* *}$, a quasi-planar self-sustaining DW equiprobably decays or transforms to a quasi-spherical DW after it leaves the tube and enters a half-space (diffraction angle $\alpha=90^{\circ}$ ).

An equation of the type (1) was carefully checked in numerous experiments; see, e.g., [6-20] in the classical formulation $\left(\alpha=90^{\circ}\right)$. This verification was motivated not only by the scientific interest in the DW diffraction process (as a phenomenon with complicated shockwave interactions) but also by considerations important for practice: diffraction is a convenient method of initiation of expanding waves in combustible mixtures, which is especially important in estimating the explosion hazard of various mixtures. The relation of the type (1) is also important because a hypothesis of the key role of the cellular structure in DW initiation and propagation was put forward, and this relation made it possible to use the parameter $a$ to estimate the critical parameters of a multifront DW, namely, the initiation energy for different types of symmetry, the geometric size of the channels for critical propagation of the DW and its modifications in channels of different configurations, the size of the charge capable of exciting detonation in 

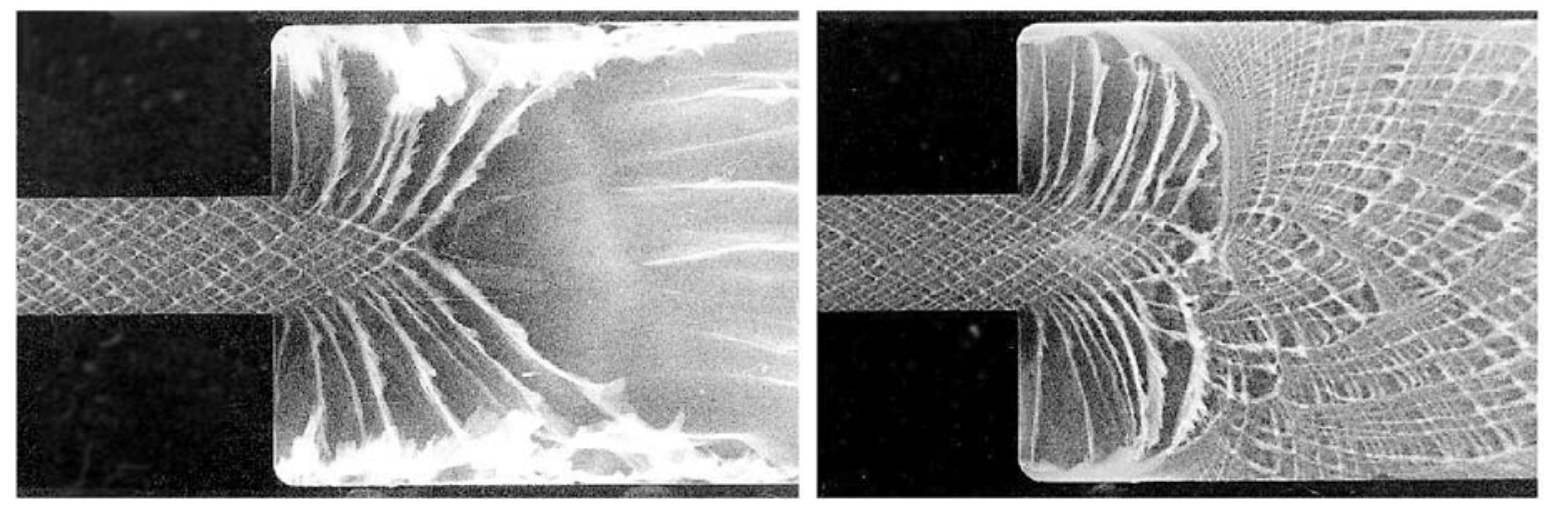

Fig. 1. Luminescence of a diffracted DW: DW decay and subsequent burning of the mixture in a high-velocity turbulent flame (left) and re-initiation of an expanding DW (right).

the combustible mixture, the critical size of unbounded gaseous charges-clouds, the critical constants characterizing the induction period, etc.

\section{Nonclassical Schemes of Wave Diffraction}

Schemes with angles $\alpha$ other than $90^{\circ}$ can be considered as nonclassical ones, because much fewer studies were performed for these cases than for the classical case with $\alpha=90^{\circ}$. Nonclassical schemes also include schemes of DW propagation in channels with complicated geometry: with branching of the channel, channel turns (smooth or sudden), and perforation of the channel walls (see, e.g., [21]). In addition, nonclassical schemes include diffraction of overdriven detonation waves, diffraction of near-critical detonation regimes (spin, marginal, or galloping detonation), diffraction of quasi-detonation (quasi-steady process of wave propagation with a velocity approximately equal to one half of the normal detonation velocity), diffraction of laminar and turbulent flames, and diffraction of accelerating flames including a particular case of the deflagration-todetonation transition (DDT).

Some diffraction schemes are presented in Fig. 2. The scheme in Fig. 2a shows the departure of a selfsustaining DW into an expanding channel with a linear $\left(y=x \tan \alpha\right.$, where $\left.\alpha=0-90^{\circ}\right)$ or curvilinear $[y=f(x)]$ boundary and preliminary generation of an overdriven DW by reducing the cross-sectional area of the flow and subsequent departure of the flow into a wide channel or into a finite-size channel, where the impact of the diffracted wave onto closely located side walls of the wide channel can play an important role in DW re-initiation. The schemes in Figs. 2b and 2c are variants of optimization of diffraction re-initiation by changing the shape of the diffracted DW [circular,

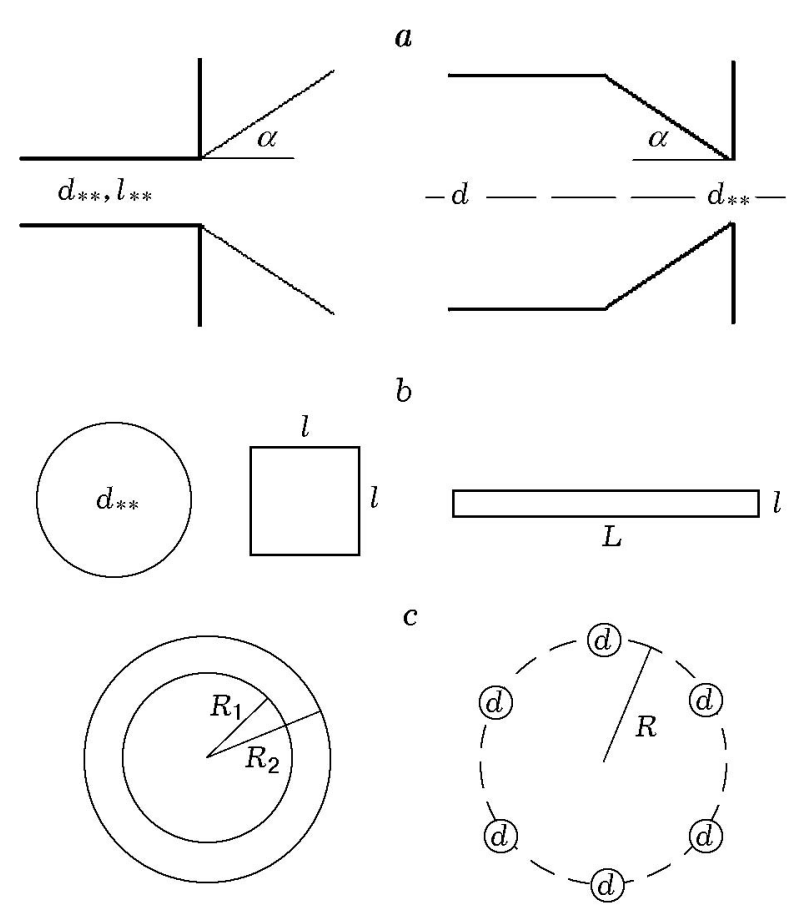

Fig. 2. Schemes of diffraction and optimization of DW re-initiation.

square, linear, or annular charges; multicharge (multipoint) scheme].

The diffraction angle, the ratio of the size of the narrow and wide channels, the degree to which the wave is overdriven, and many other factors can noticeably affect DW re-initiation under certain conditions. Some of the results of such studies were described in [21], and a review of the state-of-the-art of diffraction problems and results can be found in [22]. Recent investigations showed than relation (1) with a constant coefficient gives only an approximate estimate of the critical 

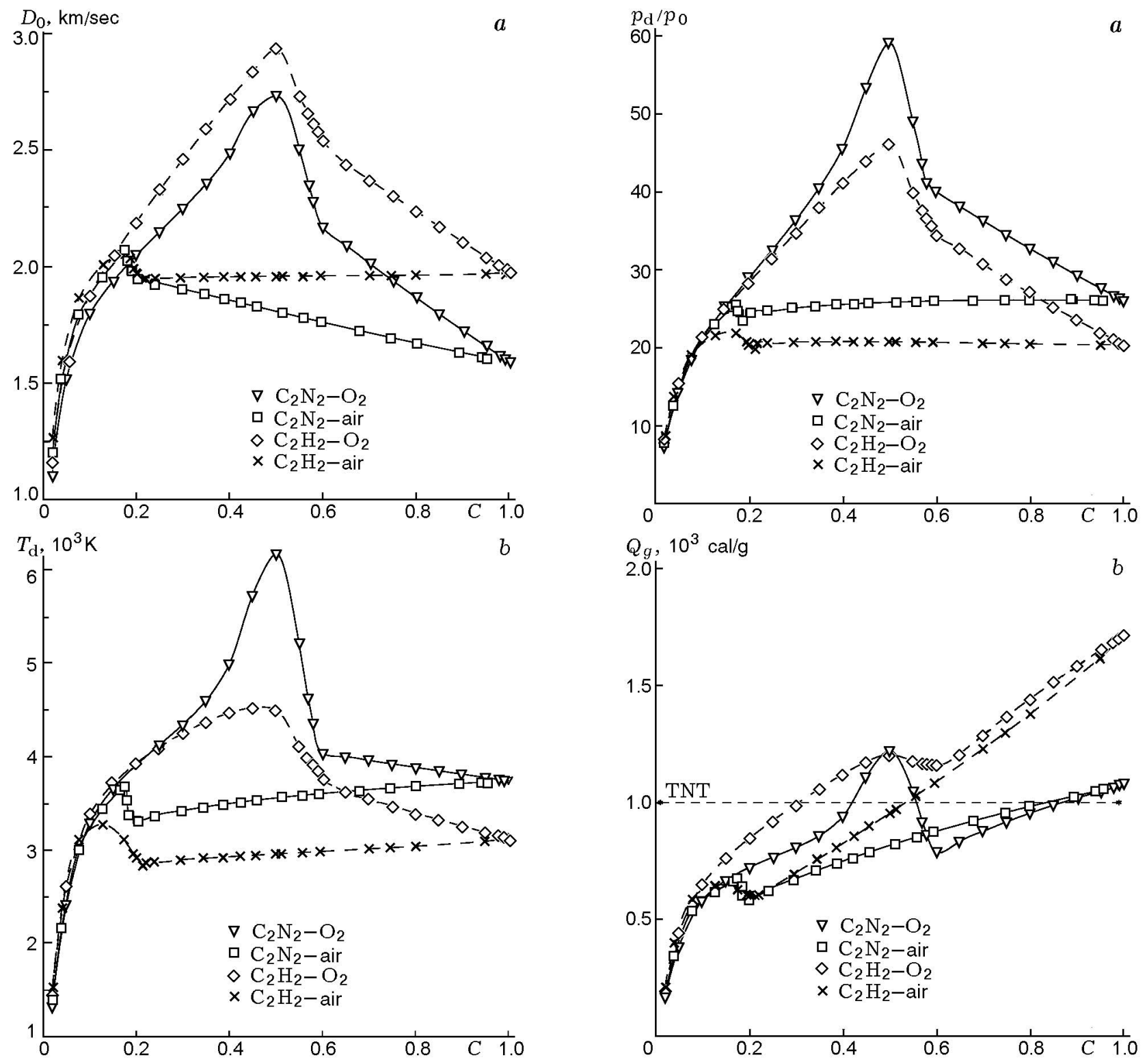

Fig. 3. DW velocity (a) and temperature of detonation products (b) versus the normalized mole fraction of the fuel for fuel-oxygen and fuel-air mixtures (calculations with allowance for carbon condensation in detonation products).

diffraction diameter; in reality, the value of $d_{* *} / a$ is not constant for various mixtures and differs at least by a factor of 2 to either side.

Numerical simulation of the diffraction of a multifront DW in a rigorous non-one-dimensional formulation is an extremely complicated task. A few codes for computing DW diffraction are only available (see [23-25]), but no critical value of the parameter $d_{* *} / a$ consistent with experimental data has been found.

Fig. 4. Pressure of detonation products (a) and energy release in the DW (b) versus the normalized mole fraction of the fuel for fuel-oxygen and fuel-air mixtures (calculations with allowance for carbon condensation in detonation products): the horizontal dashed line indicates the standard value of the TNT equivalent of $1000 \mathrm{cal} / \mathrm{g}$.

The present paper described some results of new investigations of diffraction with characteristic velocities of propagation of the chemical reaction front in the combustible mixture varied from centimeters per second (laminar flame) to kilometers per second (detonation, including strongly overdriven detonation). 


\section{Combustion and Detonation Parameters}

Most experiments were performed with mixtures of acetylene and dicyanogen with oxygen (fuels $\mathrm{H}-\mathrm{C} \equiv \mathrm{C}-\mathrm{H}$ and $\mathrm{N} \equiv \mathrm{C}-\mathrm{C} \equiv \mathrm{N}$ with triple chemical bonds). These fuels have a spectral characteristic convenient for registration: luminescence of $\mathrm{CH}$ or $\mathrm{CN}$ radicals in the blue spectral range. In addition, acetylene is known as an interesting fuel because of possible condensation of carbon in reaction products with formation of nanoparticles, fullerenes, nanotubes, etc. Similar features are also inherent in dicyanogen. The phenomena observed in various tubes and channels were recorded with the use of schlieren records, photographs of spontaneous luminescence, and pressure probes.

The parameters of combustion and detonation of these mixtures in a wide range of concentrations were computed by the "BEZOPASNOST" ("SAFETY") computer code [26]. Some results are plotted in Figs. 3-5.

The following results were obtained for an initial pressure of 1.0 atm and a temperature of $298 \mathrm{~K}$. For fuel-oxygen mixtures, the temperature of detonation products $\left(T_{\mathrm{d}}\right)$ reaches the maximum value in an equimolar mixture: $4507 \mathrm{~K}$ for acetylene and $6178 \mathrm{~K}$ for dicyanogen (which is the highest value among all gaseous fuels). For acetylene and dicyanogen, the detonation velocities are $D=2934$ and $2732 \mathrm{~m} / \mathrm{sec}$, respectively (which is close to values observed in experiments [27]), the normalized pressures of detonation products are $p_{\mathrm{d}} / p_{0}=46.1$ and 59.1 , the cell sizes are 0.08 and $0.15 \mathrm{~mm}$, and the critical diameters of diffraction reinitiation of detonation ("departure" diameter $d_{* *}$ ) are 1.0 and $1.9 \mathrm{~mm}$, respectively.

Carbon condensation starts when the acetylene and dicyanogen concentrations reach $\approx 60 \%$ in mixtures with oxygen and $\approx 20 \%$ in mixtures with air (up to $100 \%$ ). In this range of concentrations, the energy of carbon condensation plays an extremely important role in steady propagation of the detonation wave: without carbon condensation, the DW intensity is much lower. For pure acetylene and dicyanogen (rich flammability limit, without oxygen or air), the detonation velocities are 1977 and $1591 \mathrm{~m} / \mathrm{sec}$, the temperatures of combustion products are 3130 and $3746 \mathrm{~K}$, the pressure ratios are 20.4 and 26.0, and the fractions of condensed carbon in detonation products are approximately 87.5 and $89.4 \%$ of the initial amount, respectively.

The critical energy of initiation for spherical detonation is presented in Fig. 5. This plot illustrates the comparative efficiency of initiation of various mixtures (problem of safety). For fuel-oxygen mixtures

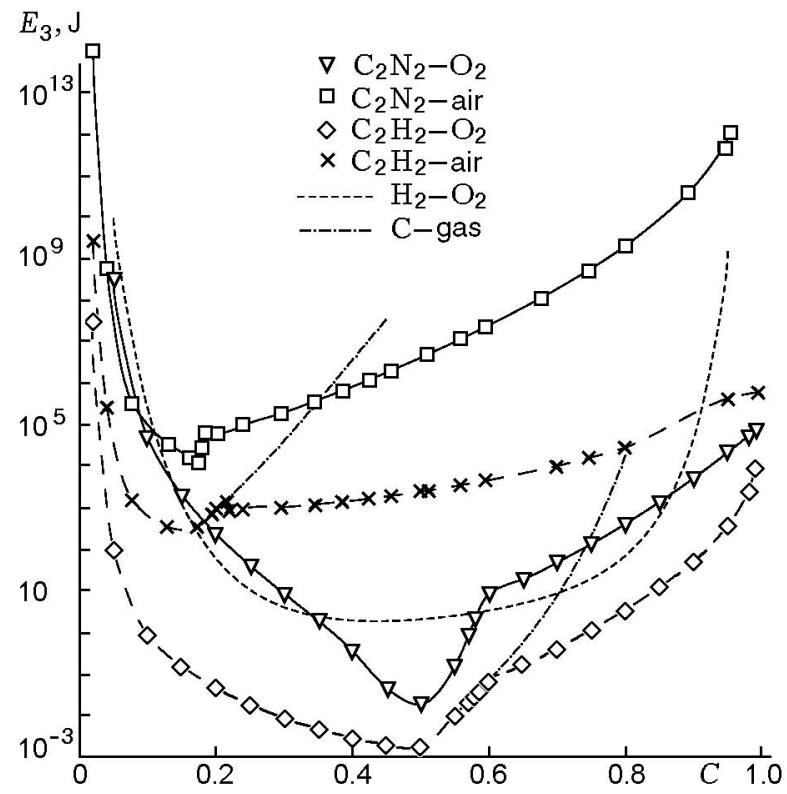

Fig. 5. Critical energy of initiation of a spherical DW versus the mole fraction of the fuel for fuel-oxygen and fuel-air mixtures (calculations with allowance for carbon condensation in detonation products).

with equimolar concentrations, the critical energies of initiation are $0.0018 \mathrm{~J}$ for acetylene and $0.018 \mathrm{~J}$ for dicyanogen. For fuel-air mixtures, the minimum energies are 350 and $12,500 \mathrm{~J}$ (cell sizes 4.7 and $13.5 \mathrm{~mm}$ ); for a stoichiometric acetylene-air mixture, the cell size is $8 \mathrm{~mm}$, the critical diameter is $144 \mathrm{~mm}$, and the critical energy of initiation is $1600 \mathrm{~J}$. The data for nonequimolar dicyanogen-oxygen mixtures are similar to those in the hydrogen-oxygen system (see Fig. 5).

\section{Basic Experimental Results}

Experimental investigations displayed versatile regimes of propagation of combustion and detonation waves both in a constant-section channel (tube) and after diffraction. We discuss only some particular regimes.

For instance, in addition to the predictable behavior of the DW after its diffraction (DW re-initiation or conversion to the combustion mode), we observed regimes of complete decay of not only detonation but of combustion as well (under certain conditions), which was manifested in complete ceasing of luminescence of detonation products. Such an effect was observed not only for a self-sustaining DW but also for an overdriven DW (some photographs are shown in Fig. 6). The nature of this effect requires additional studies.

An unconventional behavior of the wave in the case of flame diffraction is demonstrated in Fig. 7: emer- 


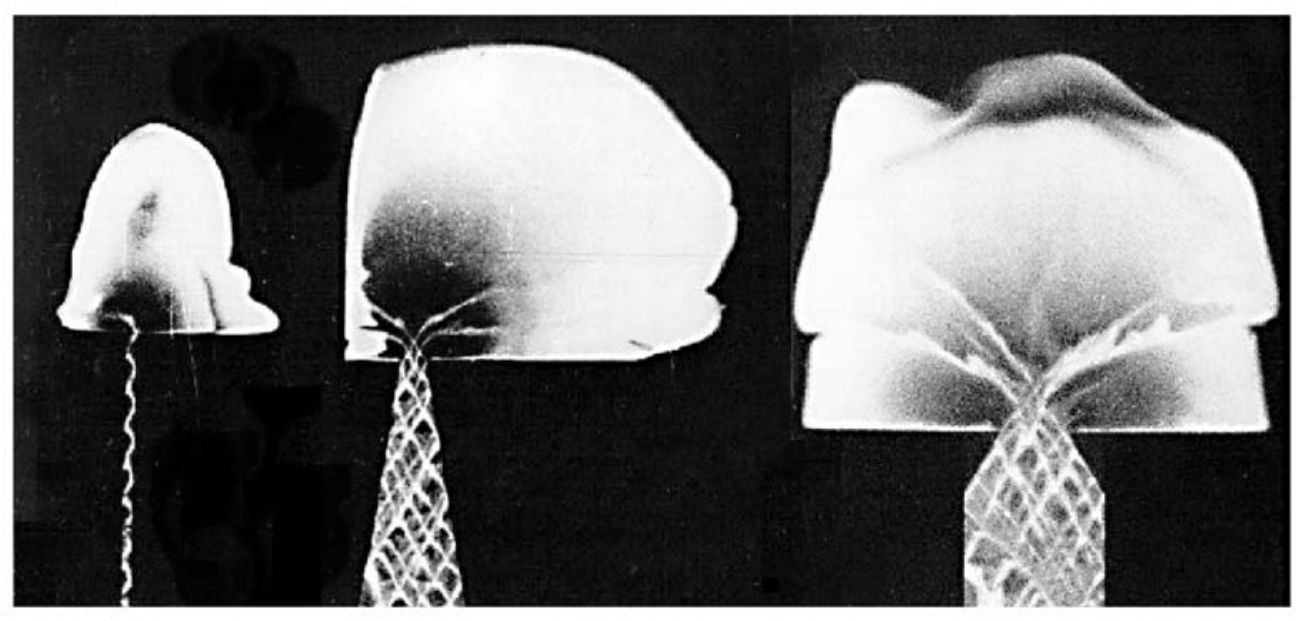

Fig. 6. Effect of complete disintegration of detonation and combustion waves due to diffraction for near-critical detonation (left frame) and even for an overdriven DW (middle and right frames).
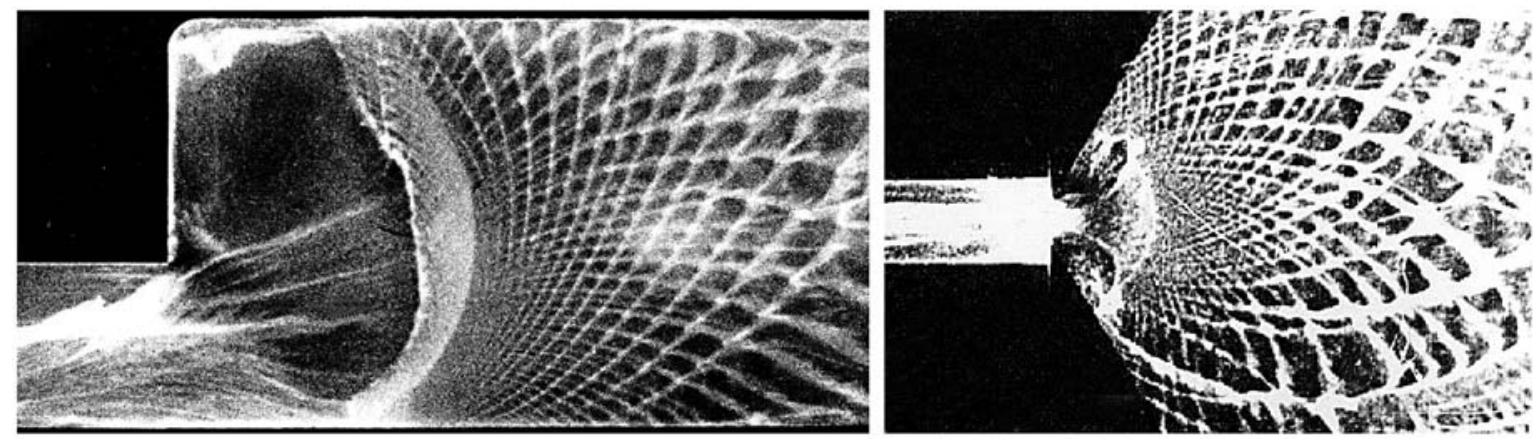

Fig. 7. DDT due to flame diffraction at $\alpha=90^{\circ}$ (left frame) and due to flame departure into an expanding channel (right frame).

gence of detonation in a wide channel after flame expansion. Such a situation occurs if the DDT point is located in the vicinity of the diffraction section. The latter can be ensured by varying the initiator energy [the critical energy of ignition of the mixture is always lower (by several orders) than the critical energy of DW initiation], composition of the mixture, initial pressure, length of the initiation section, etc. DW initiation due to flame diffraction is of particular interest because the DDT ensures the maximum possible pressures (owing to a strongly overdriven DW), which are much higher than the pressures of detonation products of a self-sustaining DW. Approximately half a century ago [28], the DDT process near the closed end of a tube (owing to variation of the composition of the mixture) destroyed a metal wall $10 \mathrm{~mm}$ thick! This is a very dangerous situation, which cannot be ignored in modeling the origination and development of the emergency hot spot in space with various obstacles. Another viewpoint is that optimization of initiation processes can be efficiently controlled if we learn to control the position of the DDT point.

An unusual effect of the deflagration-to-detonation transition near the diffraction section is illustrated in Fig. 8, which shows the critical pressure of the mixture $48 \% \mathrm{C}_{2} \mathrm{~N}_{2}+52 \% \mathrm{O}_{2}$ for successful re-initiation of the detonation wave owing to diffraction (DW departure from a tube with a diameter $d=6 \mathrm{~mm}$ to a tube with $d=44 \mathrm{~mm}$ ) versus the length $L$ of the initiation section of the tube. For $L>400 \mathrm{~mm}$, the conditions of diffraction re-initiation become independent of the length of the initiation section. The reason is that the DDT point is localized in the initiation section ahead of the diffraction section, and the conventional DW diffracts in this case. The situation with $L<400 \mathrm{~mm}$, however, dis- 


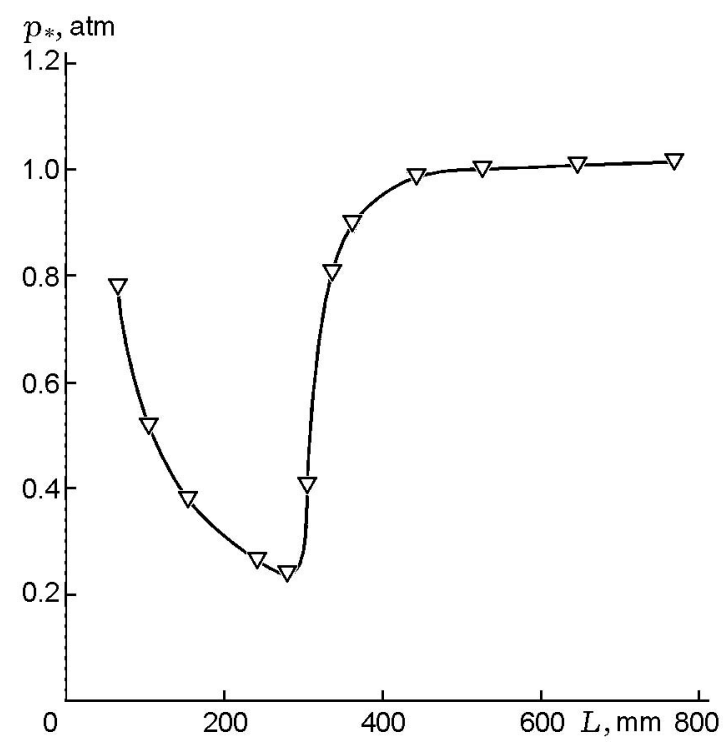

Fig. 8. Critical pressure in the mixture $48 \% \mathrm{C}_{2} \mathrm{~N}_{2}+52 \% \mathrm{O}_{2}$ as a function of the length of the initiation section of the tube.

plays an unconventional behavior: successful diffraction re-initiation of the detonation wave at lower pressures, with a certain optimal value $p_{*}$. The nature of this effect is associated with the diffraction section approaching the DDT point (by means of length variation), and a powerful wave generated by the DDT obviously provides DW re-initiation not only at the optimal value of pressure but also at lower pressures. If the DDT point goes far beyond the diffraction section and moves away from the optimal position, the pressure has to be again increased from $p_{*}$ to reach DW re-initiation.

It should be emphasized that the unconventional regimes of diffraction considered in the present paper can serve as good test cases for future numerical simulations. Such simulations have not yet been performed.

\section{CONCLUSIONS}

A large variety of regimes of propagation of combustion and detonation waves with diffraction is observed. Controlling the position of the deflagrationto-detonation transition point is found to be a highly efficient method for optimizing the initiation processes.

This work was supported by the Russian Foundation for Basic Research (Grant No. 05-01-00129), Foundation "Leading Scientific Schools of Russia" (Mechanics of Shock and Detonation Processes, Grant NSh858.2006.1), and INTAS Foundation (Grant No. 03-51$3332)$.

\section{REFERENCES}

1. C. Campbell, "The propagation of explosion waves in gases contained in tubes of varying cross-section," J. Chem. Soc., 2483-2498 (1922).

2. P. Laffitte, "On the propagation of a spherical explosion wave," Com. Rend. Acad. Sci., 177, 178-180 (1923).

3. Ya. B. Zel'dovich, S. M. Kogarko, and N. N. Simonov, "Experimental study of spherical gas detonation," $Z h$. Tekh. Fiz., 26, No. 8, 1744-1769 (1956).

4. B. V. Voitsekhovskii, V. V. Mitrofanov, and M. E. Topchiyan, Detonation Front Structure in Gases [in Russian], Izd. Sib. Otd. Akad. Nauk SSSR, Novosibirsk (1963)

5. V. V. Mitrofanov and R. I. Soloukhin, "Diffraction of a multifront detonation wave," Dokl. Akad. Nauk SSSR, 159, No. 5, 1003-1006 (1964).

6. S. M. Kogarko, "Possibility of detonation of gas mixtures in conical tubes," Izv. Akad. Nauk SSSR, Otd. Khim. Nauk, No. 4, 419-426 (1956).

7. R. A. Strehlow, A. A. Adamczyk, and R. J. Stiles, "Transient studies of detonation waves," Astronaut. Acta, 17, Nos. 4-5, 509-527 (1972).

8. R. A. Strehlow and R. J. Salm, "The failure of marginal detonations in expanding channels," Acta Astronaut., 3, No. 11, 983-994 (1976).

9. D. H. Edwards, G. O. Thomas, and M. A. Nettleton, "The diffraction of a planar detonation wave at an abrupt area change," J. Fluid Mech., 95, No. 1, 79-96 (1979).

10. A. A. Vasil'ev and V. V. Grigor'ev, "Critical conditions for gas detonation in sharply expanding channels," Combust., Expl., Shock Waves, 16, No. 5, 579585 (1980).

11. R. Knystautas, J. H. S. Lee, and C. M. Guirao, "The critical tube diameter for detonation failure in hydrocarbon-air mixtures," Combust. Flame, 48, 63-83 (1982).

12. D. Desbordes and M. Vachon, "Critical diameter of diffraction for strong plane detonations," in: J. R. Bowen, J.-C. Leyer, and R. I. Soloukhin (eds.), Progress in Astronautics and Aeronautics, Vol. 106: Dynamics of Explosion, New York (1986), pp. 131-143.

13. W. B. Benedick, R. Knystautas, and J. H. Lee, "Large-scale experiments on the transmission of fuelair detonations from two-dimensional channels," in: I. R. Bowen, N. Manson, A. K. Oppenheim, and R. I. Soloukhin (eds.), Progress in Astronautics and Aeronautics, Vol. 94: Dynamics of Shock Waves, Explosions and Detonations, New York (1983), pp. 546-556.

14. Y. K. Liu, J. H. Lee, and R. Knystautas, "Effect of geometry on the transmission of detonation through an orifice," Combust. Flame, 56, 215-225 (1984). 
15. J. O. Moen, A. Sulmistras, G. O. Thomas, et al., "The influence of cellular regularity on the behaviour of gaseous detonations," in: J. R. Bowen, J.-C. Leyer, and R. I. Soloukhin (eds.), Progress in Astronautics and Aeronautics, Vol. 106: Dynamics of Explosion, New York (1986), pp. 220-243.

16. A. A. Vasil'ev, "Initiation of a gas detonation with a spatial source distribution," Combust., Expl., Shock Waves, 24, No. 2, 232-237 (1988).

17. A. A. Vasil'ev, "Spatial excitation of a multifront detonation," Combust., Expl., Shock Waves, 25, No. 1, 104108 (1989).

18. A. A. Vasil'ev, "Gas detonation propagation with simultaneous change in tube section and mixture composition," Combust., Expl., Shock Waves, 21, No. 2, 262265 (1985).

19. N. V. Bannikov and A. A. Vasil'ev, "Plane initiation of a detonation," Combust., Expl., Shock Waves, 29, No. 3, 409-414 (1993).

20. A. A. Vasil'ev, "Near-critical regimes of gas detonation," Doct. Dissertation in Phys.-Math. Sci., Inst. of Hydrodynamics, Sib. Div., Russian Acad. of Sci. (1995).

21. A. A. Vasil'ev, "Modes of a detonation and highspeed burning in channels with perforated walls," in: V. Molkov (ed.), Fire-and-Explosion Hazard of Substances and Venting of Deflagrations, Proc. of the Second Int. Seminar, Inst. for Fire Protection, Moscow (1998), pp. 582-592.

22. A. A. Vasil'ev et al., "The basic results of reinitiation processes in diffracting multifront detonations. Part I," Eurasian Chem.-Technol. J., 5, No. 4 (2003).
23. K. Hiramatsu, T. Fujiwara, and S. Taki, "A computational study of transmission of gaseous detonation to unconfined space," in: Proc. 20th Symp. (Int.) on Combustion, Pittsburgh (1984).

24. M. Fisher, E. Pantow, and T. Kratzel, "Propagation, decay and re-ignition of detonations in technical structures," in: G. Roy, S. Frolov, K. Kailasanath, and N. Smirnov (eds.), Gaseous and Heterogeneous Detonations. Science to Applications, ENAS Publ., Moscow (1999), pp. 197-212.

25. B. Khasainov, C. Priault, H.-N. Presles, and D. Desbordes, "On the mechanism of transition of self-sustained detonation from a tube to a half-space through an annular orifice with central obstacle," in: Proc. 18th Int. Colloquium on the Dynamics of Explosions and Reactive Systems (July 29-August 03, 2001, Seattle), Univ. Washington. CD ISBN 0-9711740-0-8, No. 096.

26. A. A. Vasil'ev, A. I. Valishev, V. A. Vasil'ev, and L. V. Panfilova, "Combustion and detonation characteristics of hydrazine and its methyl derivatives," Combust., Expl., Shock Waves, 36, No. 3, 358-373 (2000).

27. G. Munday, A. R. Ubbelohde, and I. F. Wood, "Marginal detonation in cyanogen/oxygen mixtures," Proc. Roy. Soc. A, 306, No. 1485, 179-184 (1968).

28. S. M. Kogarko, "Pressure at the end of a tube with unsteady fast combustion," Zh. Tekh. Fiz., 28, No. 9, 2041-2045 (1958). 